\title{
iCluster: a Self-Organizing Overlay Network for P2P Information Retrieval
}

\author{
Paraskevi Raftopoulou and Euripides G.M. Petrakis \\ Department of Electronic and Computer Engineering, \\ Technical University of Crete (TUC), \\ Chania, Crete, GR-73100, Greece \\ \{paraskevi, petrakis\}@intelligence.tuc.gr
}

\begin{abstract}
We present iCluster, a self-organizing peer-to-peer overlay network for supporting full-fledged information retrieval in a dynamic environment. iCLUSTER works by organizing peers sharing common interests into clusters and by exploiting clustering information at query time for achieving low network traffic and high recall. We define the criteria for peer similarity and peer selection, and we present the protocols for organizing the peers into clusters and for searching within the clustered organization of peers. iCLUSTER is evaluated on a realistic peer-to-peer environment using real-world data and queries. The results demonstrate significant performance improvements (in terms of clustering efficiency, communication load and retrieval accuracy) over a state-of-the-art peerto-peer clustering method. Compared to exhaustive search by flooding, iCLUSTER exchanged a small loss in retrieval accuracy for much less message flow.
\end{abstract}

\section{Introduction}

Information sharing in a peer-to-peer (p2p) network requires searching in a distributed collection of peers [1]. Distributed Hash Tables (DHTs) [2,3] and Semantic Overlay Networks (SONs) $[4,5]$ are common solutions to the problem of fast information search in $\mathrm{p} 2 \mathrm{p}$ networks. DHTs provide fast lookup mechanisms facilitating information search over the network assuming that each peer is connected to other peers and is responsible for a part of the distributed index. SONs provide an alternative solution to the problem of decentralized indexing by relaxing the requirement of strict peer connectivity imposed by DHTs: peers are virtually linked together (forming clusters) based on the likelihood to contain similar content. The problem of finding the most relevant resources is then reduced to the one of locating clusters of peers similar to the query.

We present iCLUSTER, an approach towards efficient organization of $\mathrm{p} 2 \mathrm{p}$ networks into SONs that supports Information Retrieval (IR) functionality: iCluster is automatic (requires no intervention by the user), general (requires no previous knowledge of the peers' contents and works for any type of text contents), adaptive (adjusts to dynamic changes of the network contents), efficient 
(query processing is faster than existing solutions in the literature) and accurate (achieves high recall outperforming current approaches).

Recent work on SONs by Loser [6] suggests combining information from all layers for scoring the peers. Spripanidkulchai [7] introduced the notion of peer clustering based on similar interests rather than similar documents. Edutella [8] uses metadata to arrange super-peers into the so called HyperCup topology [9]. Finally, Lu [10] suggests using content-based information to route query messages to a subset of neighboring peers. However, the work referred above assumes one interest per peer (peer specialization). Klampanos [11] proposed an approach for clustering peers holding information on more that one topics. Parreira [12] introduces the notion of "peer-to-peer dating" for allowing peers to decide which connections to create and which to avoid, based on various usefulness estimators. Additional work on peer organization using SONs is based on the idea of "small world networks" [13,14]. Schmitz [5] assumes that peers share concepts from a common ontology, and this information is used for organizing peers into communities (small worlds) with similar concepts.

iCLUSTER extends the idea of peer organization in small world networks by Schmitz [5] in the following ways: (a) Peers contribute documents in the network (rather than concepts from an ontology). To that end, peers are represented in the network by their interests (in fact document descriptions derived from their content by automatic text processing). Accordingly, query processing imposes document search operations over the network. (b) This organization allows for peers with multiple and dynamic interests (not known in advance). (c) iCLUSTER proposes new rewiring protocols for achieving dynamic (on the fly) organization of peers in clusters and also, effective information search in the derived clustered organization of peers.

The rest of this paper is organized as follows: iCLUSTER architecture and protocols are discussed in Sect. 2, experimental results are presented and discussed in Sect. 3, followed by conclusions and issues for future research in Sect. 4.

\section{2 iCluster}

Each peer is characterized by the content of the documents it contributes to the network. Peers with similar interests are grouped together into clusters. Peers may have more than one interests and belong to more than one clusters. Each peer maintains a routing index holding information for short- and long-range links to other peers:

short-range links correspond to intra-cluster information (i.e., links to peers with similar interests).

long-range links correspond to inter-cluster information (i.e., links to peers belonging to different clusters and thus, having different interests).

Entries in the routing index are of the form $\left(i p\left(p_{j}\right), c_{j k}\right)$, where $i p\left(p_{j}\right)$ is the IP address of peer $p_{j}$ the link points to and $c_{j k}$ is the $k$-th interest of $p_{j}$. The number of routing indices maintained by a peer equals the number of peer's 
interests. Peers may merge or split their interests by merging or splitting their corresponding routing indices.

\subsection{Peer Similarity}

Initially, each peer organizes its documents into groups by applying a document clustering algorithm [15]. The documents of a peer may belong to more than one clusters (i.e., the peer may have more than one interests). Documents are represented by term vectors, and each cluster $k, k \in\left[1, L_{i}\right]$, is represented by its centroid $c_{i k}$ (i.e., the mean vector of the vector representations of the documents it contains). Each peer $p_{i}$ is represented by the list $\left\{c_{i 1}, c_{i 2}, \ldots, c_{i L_{i}}\right\}$ with the cetroids of its clusters.

A peer $p_{i}$ can be related to another peer $p_{j}$ by virtue of more than one interests. The similarity between peers $p_{i}$ and $p_{j}$ with respect to interest $k$ of $p_{i}$ is defined as

$$
\mathrm{S}_{i j}^{k}=S^{k}\left(p_{i}, p_{j}\right)=\max _{\forall y}\left\{\operatorname{Sim}\left(c_{i k}, c_{j y}\right)\right\},
$$

where $c_{i k}, c_{j y}$ are the interests of $p_{i}$ and $p_{j}$, and $\operatorname{Sim}\left(c_{i k}, c_{j y}\right)$ is the similarity between their centroid document vectors. The overall similarity between two peers is defined as the maximum similarity over all pairs of cluster centroids:

$$
S\left(p_{i}, p_{j}\right)=\max _{\forall x, y}\left\{\operatorname{Sim}\left(c_{i x}, c_{j y}\right)\right\}
$$

Finally, the similarity between a document (or query) $d$ and a peer $p_{i}$ is defined as the maximum similarity between the document (or query) and the peer's interests (centroids):

$$
\operatorname{sim}\left(d, p_{i}\right)=\max _{\forall x}\left\{\operatorname{Sim}\left(d, c_{i x}\right)\right\}
$$

\section{2 iCluster Protocols}

The main idea behind iCLUSTER is to let peers self-organize into SONs, and then, search for similar answers (documents) by addressing the most similar clusters to a given query. The protocols regulating peer join, generation of peer clusters, and query processing in iCLUSTER are discussed next.

Peer Join: When a peer $p_{i}$ connects to the network, it computes its description $\left\{c_{i 1}, c_{i 2}, \ldots, c_{i L_{i}}\right\}$. For each interest $c_{i k}$ in its description, $p_{i}$ maintains a routing index $R I_{i k}$, which is constructed as follows: $p_{i}$ issues a request to the network that, through a random walk, collects in $R I_{i k}$ the IP addresses and descriptions from $\lambda$ (randomly) visited peers, which form the initial neighborhood $\nu_{i k}$ of $p_{i}$. These (randomly selected) links will be refined according to $p_{i}$ 's $k$-th interest, using the peer organization protocol below. 
Peer Organization: Peer organization proceeds by establishing new connections and by discarding old ones, producing this way groups of peers with similar interests. Each peer $p_{i}$ periodically initiates a rewiring procedure. For each interest $k, p_{i}$ computes the intra-cluster similarity $N S_{i k}$ (as a measure of cluster cohesion) as

$$
N S_{i k}=\frac{1}{\left|\nu_{i k}\right|} \cdot \sum_{\forall p_{j} \in \nu_{i k}} \mathrm{~S}_{i j}^{k},
$$

where $\left|\nu_{i k}\right|$ is the number of peers in the neighborhood of $p_{i}$ with respect to interest $k$. If $N S_{i k}$ is greater than a threshold $\theta$ ( $\theta$ is user defined), then $p_{i}$ does not need to take any further action, since it is surrounded by peers with similar interests. Otherwise, $p_{i}$ initiates a cluster refinement process by issuing FindNodes $=\left(i p\left(p_{i}\right), c_{i k}, P, t_{F}\right)$ message, where $i p\left(p_{i}\right)$ is the IP address of $p_{i}$, $c_{i k}$ is the centroid corresponding to $k$-th interest of $p_{i}$ and $t_{F}$ is the time-tolive (TTL) of the message ( $t_{F}$ is user defined). A peer $p_{j}$ receiving the message computes the similarity between its interest $c_{j y}$ with interest $c_{i k}$ in FindNodES message, appends to $P$ the interest resulted in the maximum similarity value, reduces $t_{F}$ by 1 and forwards FindNoDES message to its neighbors. When $t_{F}=$ 0 , FindNodes message is sent back to the initial sender $p_{i}$. The message is forwarded with equal probability either to (i) a number $m$ of randomly chosen peers contained in $p_{j}$ 's routing index, or (ii) to the $m$ peers most similar to $p_{i}$ (the sender of the message). The rationale of applying both forwarding solutions at the same time is not only to connect $p_{i}$ directly to similar peers, but also indirectly, by enabling propagation of the forwarding message to other similar peers through non-similar peers in the neighborhood of $p_{i}$. Figure 1 summarizes the steps of the above rewiring process.

A peer $p_{j}$ receiving FINDNODES message collects information about new peers with similar interests, and appends it in its routing index $R I_{j \kappa}$ by replacing old short-range links corresponding to less similar peers with new links corresponding to more similar peers. Additionally, $p_{j}$ collects information about peers with non-similar interests in $R I_{j \kappa}$ updating its long-range links.

Query Processing: Queries are issued by free text or keywords and are formulated as term vectors. The peer issuing the query initiates a QueRY $=\left(q, t_{q}\right)$ message, where $q$ is the query vector and $t_{q}$ is the TTL of the message $\left(t_{q}\right.$ is user defined). The initiator $p_{i}$ of the message compares $q$ against its interests and decides for the forwarding of the message to some or all of its neighbors according to the query routing strategy that follows. Similarly, peers receiving a QUERY message compare $q$ against their interests and forward the message to neighboring peers.

A forwarding peer $p_{j}$ compares $q$ against its interests and forwards $q$ to its short-range links (i.e., broadcasts the message to its neighborhood) if $\operatorname{sim}\left(q, p_{j}\right) \geq \theta$. Otherwise, $p_{j}$ forwards $q$ to $m$ peers, that are the most similar peers to $q$ (fixed forwarding). At each step of the forwarding procedure, $t_{q}$ is reduced by 1 . 
Procedure Rewiring $\left(p_{i}, k, t_{F}, \theta, m\right)$

A procedure initiated by a peer $p_{i}$ whenever its neighborhood similarity $N S_{i k}$ drops below a predefined threshold $\theta$.

input: peer $p_{i}$ with interest $c_{i k}$ and routing index $R_{i k}$ output: updated routing index $R_{i k}$

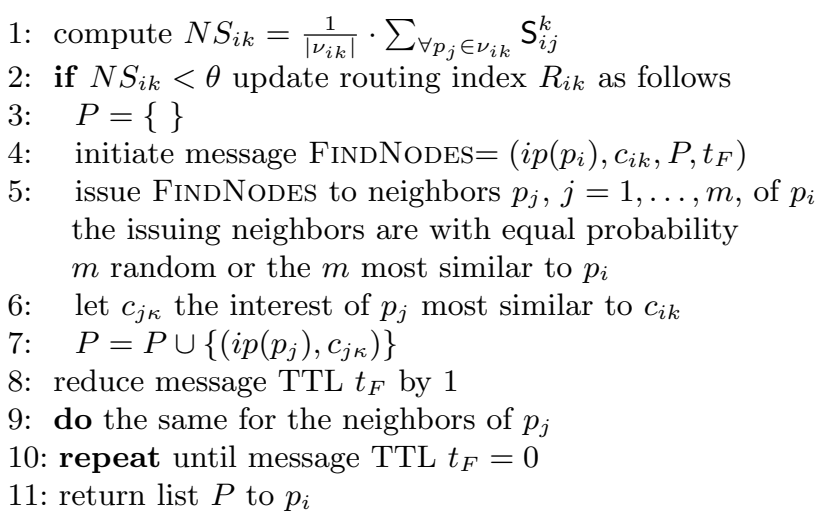

Fig. 1. Peer organization procedure.

Apart from query forwarding, each peer $p_{j}$ receiving $q$ applies the following procedure for retrieving documents similar to $q$. The peer compares $q$ against its interests and if $\operatorname{sim}\left(q, p_{j}\right) \geq \theta$ the peer matches $q$ against its locally stored content to retrieve similar documents. Pointers to these documents are sent to the initiator of the query $p_{i}$. When this process is completed, $p_{i}$ produces a list $R$ with results of the form $\langle d, \operatorname{Sim}(q, d)\rangle$, where $d$ is a pointer to a document and $\operatorname{Sim}(q, d)$ is the similarity between $q$ and $d$. The candidate answers are ordered by similarity to the query and returned to the user. Figure 2 summarizes the steps of the query processing algorithm.

\subsection{Discussion}

iCLUSTER is highly dynamic as it allows for random insertions or deletions of new documents in existing peers. Peers recompute their interests when their document collection has fairly changed. iCLUSTER is based solely on local interactions, requiring no previous knowledge of the network structure or of the overall content in the network. Each peer initiates a rewiring procedure every time the overall similarity of the peers in its neighborhood (intra-cluster similarity) drops below a predefined threshold. The cost of this organization results in extra message traffic, which increases with threshold $\theta$. However, this extra message traffic is traded for faster and more efficient search at query time. 


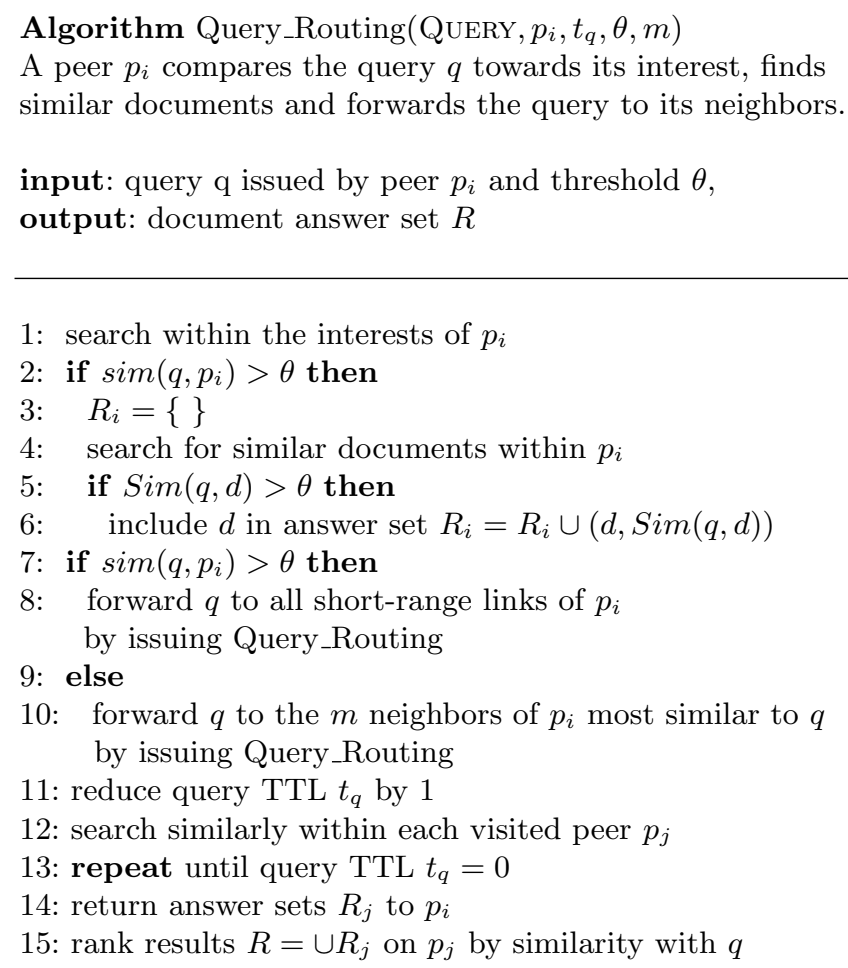

Fig. 2. Query routing algorithm.

iCLUSTER maintains a fixed number of long-range links (i.e., links to other clusters) in the routing indices of the peers in addition to short-range links. This prevents clusters from becoming isolated and thus inaccessible by other clusters.

Methods such as [8,5] assuming one interest per peer (specialization assumption) might not perform well under this setting: the description of a peer would either reflect the contents of its strongest interest (e.g., the one with more documents) ignoring all other interests, or result in a single cluster corresponding to the averaging over the entire document collection of the peer. This in turn, would result in poor retrieval performance as queries (even very specific ones) will be addressing highly incoherent clusters of peers. In iCLUSTER, each peer identifies its interests by applying a local clustering process.

\section{Evaluation}

The experiments are designed to demonstrate the superiority of the proposed iCLUSTER protocols over (a) a state-of-the-art approach for peer organization and retrieval [5] and (b) exhaustive search by flooding [16]. 


\subsection{Experimental Set-Up}

iCLUSTER has been tested on a subset of the OHSUMED TREC ${ }^{1}$ collection with 30,000 medical articles and on the TREC $6^{2}$ data set with 556,078 documents. Each OHSUMED document belongs to one out of 10 classes while, each document in the TREC6 collection belongs to one out of 100 classes.

The network consists of 2,000 peers. Initially, each peer is assigned documents from one class (i.e., initially each peer has one interest). Each peer maintains one routing index with links to other peers ( $10 \%$ are long-range links).

Each peer periodically tries to find better neighbors by initiating the rewiring procedure. The base unit for time used is the period $t$. The start of the rewiring procedure for each peer is randomly chosen from the time interval $[0,4 K \cdot t]$ and its periodicity is randomly selected from a normal distribution of $2 K \cdot t$ for each peer separately. We start checking the network at time $4 K \cdot t$, when all peers have initiated at least once the rewiring procedure.

We experimented with different values of similarity threshold $\theta$, message forwarding TTL $t_{F}$ and query forwarding TTL $t_{q}$. In the following, we show how the critical values characterizing the network vary over time. We considered 5 different initial network topologies and for each topology the results were averaged over 5 runs.

\subsection{Performance Measures}

The performance of iCLUSTER is mainly evaluated in terms of peer organization, communication load and accuracy of retrieval (recall). The (weighted) clustering coefficient $\overline{w \gamma}$ is one of the common metrics $[17,18]$ used to describe how well the peers are organized into groups with similar interests and is defined as:

$$
\overline{w \gamma}=\frac{1}{N} \sum_{i=1}^{N}\left(\frac{1}{\lambda(\lambda-1)} \sum_{\forall p_{j}, p_{k} \in \nu_{i}, p_{k} \in \nu_{j}} S\left(p_{j}, p_{k}\right)\right)
$$

The network load of a method is measured by the number of messages exchanged by the peers during rewiring or querying. In turn, the accuracy of retrieval is evaluated using recall (i.e., percentage of qualifying answers retrieved with respect to the total number of qualifying answers in the network). An organization (or search) strategy is better than another if it achieves better clustering coefficient (or retrieval accuracy) for less communication load.

\subsection{Peer Organization}

To evaluate the clustering effectiveness of iCLUSTER, we monitored how $\overline{w \gamma}$ varies over time for different values of $\theta$ and $t_{F}$. After a few iterations (after $9 \mathrm{~K}$. $t), \overline{w \gamma}$ stabilizes to 0.21 for the OHSUMED and 0.55 for the TREC6 data set. The

\footnotetext{
${ }^{1}$ http://trec.nist.gov/data/t9_filtering.html

2 http://boston.lti.cs.cmu.edu/callan/Data/
} 
variation in $\overline{w \gamma}$ is due to the variation in the number of document classes in the two data sets. Stability is achieved as peers are surrounded eventually by other peers with similar interests (i.e., $N S>\theta$ ). The experiments demonstrate that the values of $\overline{w \gamma}$ are slightly influenced by $\theta$ (less than $3 \%$ ). Additionally, only a small number of organization messages are initially needed and are reduced to 0 after time $6 K \cdot t$, when the network becomes coherent.

Additional experiments indicate that the network converges faster for higher values of $t_{F}$ (i.e., high values of $t_{F}$ address peers far apart from the peer originating the process). Although $\overline{w \gamma}$ do not vary significantly with $t_{F}$, the communication overhead increases by $86 \%$ (i.e., when $t_{F}$ increases from 4 to 7 ), leading us to choose $t_{F}=4$ for our setting.

The experiments above demonstrate that the rewiring protocol of iCLUSTER results in a effective peer organization at the expense of small communication load. The rewiring similarity threshold $\theta$ affects clustering cohesion, while the rewiring TTL parameter $t_{F}$ has minimal effects on the convergence time of the network.

\subsection{Performance of Retrieval}

The purpose of this set of experiments is to evaluate the performance of the proposed query routing protocol as a function of (i) recall, (ii) communication overhead incurred by a query and (iii) recall per search message. We also examine the dependance of recall and communication overhead on $t_{q}, \theta$ and $t_{F}$. The plots below correspond to measurements on the OHSUMED data set (TREC6 produced similar results).

Figure 3 illustrates how recall varies with time for various values of $\theta$. When no similarity structure is imposed in the network $(4 K \cdot t)$, the queries are flooded over the network reaching recall as high as 0.8 . However, this value of recall is achieved for large communication overhead (1,200 messages per query). When the network becomes organized into cohesive clusters (after time $9 K \cdot t$ ), iCLUSTER achieves the same high values of recall for much less communication overhead (500 messages per query).

As shown in Fig. 3, $\theta=0.6$ achieves the highest recall on an organized network (after time $9 K \cdot t$ ). For lower values of $\theta$, there are many links from each peer towards non-similar peers, since the clusters are not coherent enough. For higher values of $\theta$, the clusters are coherent but it becomes difficult for a query to be forwarded to similar clusters of peers through other non-similar peers. The optimal value of $t_{F}$ achieving the best recall is 6 .

Figure 4 shows the dependence of recall and communication load incurred by the query (in number of messages sent) on $t_{q}$. Obviously, recall increases with $t_{q}$ as more peers are receiving $q$, but communication load increases as well. For $t_{q}=4$ or 6 the recall achieved is very low. Notice that $t_{q}=10$ achieved almost $19 \%$ better recall (approaching recall 1) than $t_{q}=8$ at the expense of $53 \%$ more communication load. Based on these observations, the suggested value of $t_{q}$ is 8. Although $t_{q}=8$ is relatively high, the communication overhead is low as iCLUSTER applies selective propagation of query messages to qualifying peers. 


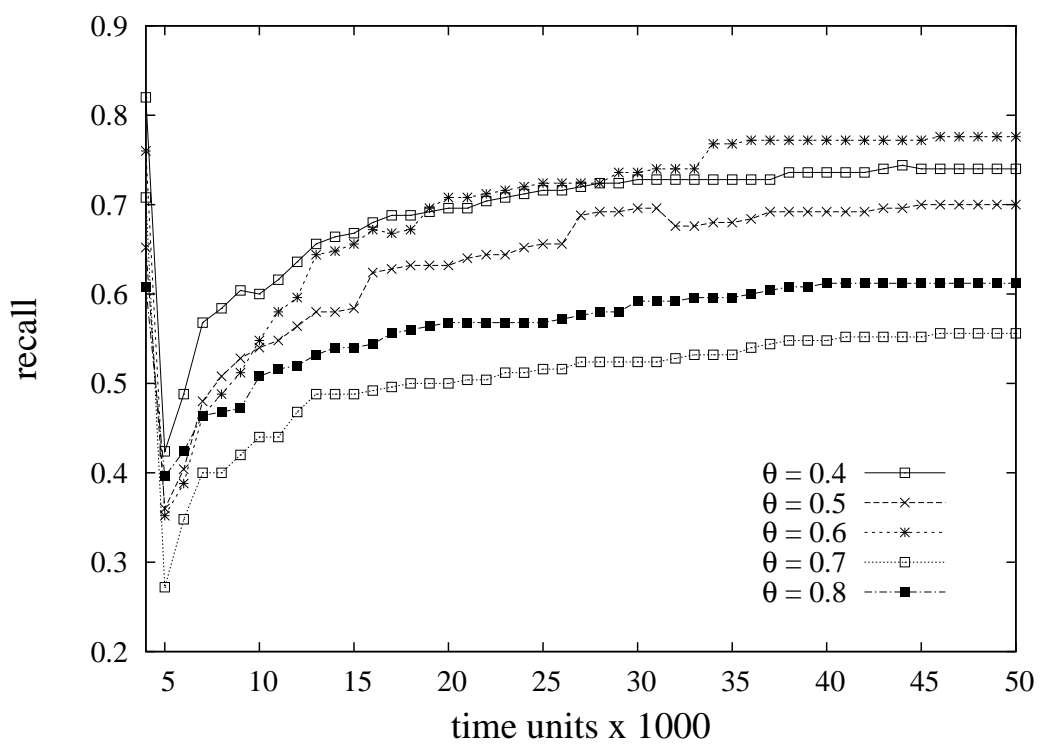

Fig. 3. Recall as a function of time for various values of $\theta$.

The experiments above showed that iCLUSTER achieves high values of recall for less communication load when the network becomes organized into cohesive clusters. We examined the dependance of the retrieval performance on the rewiring process and on $t_{q}$, and we suggested optimal values for the parameters (i.e., achieving high recall with small communication overhead).

\subsection{Comparison to Other Methods}

The following methods are implemented and compared:

- iCluster, the method proposed in this work for $\theta=0.6, t_{F}=6$ and $t_{q}=8$.

- Query flooding, the method implemented by many p2p systems (e.g., Gnutella). It assumes no special network structure and the query is flooded over the network. For comparison with iCLUster we set $t_{q}=8$.

- The peer organization approach proposed by Schmitz [5], using $\theta=0.6$, $t_{F}=6$ and $t_{q}=8$ to have results that are comparable with iCLUSTER.

Notice that $\overline{w \gamma}$ is close to 0 for flooding, indicating no organization of peers into clusters. Compared to [5], iCLuster results in higher value of $\overline{w \gamma}(0.21$ as opposed to 0.08) and therefore, better clustering quality.

Figure 5 shows how recall varies over time for all three approaches. The flooding approach achieves recall 0.85 as it searches the network almost exhaustively imposing high communication overhead. As Fig. 5 indicates, prior to imposing any similarity structure in the network (before time $6 K \cdot t$ ), iCLUSTER and [5] achieves recall as high as 0.8 . However, notice the high communication overhead 


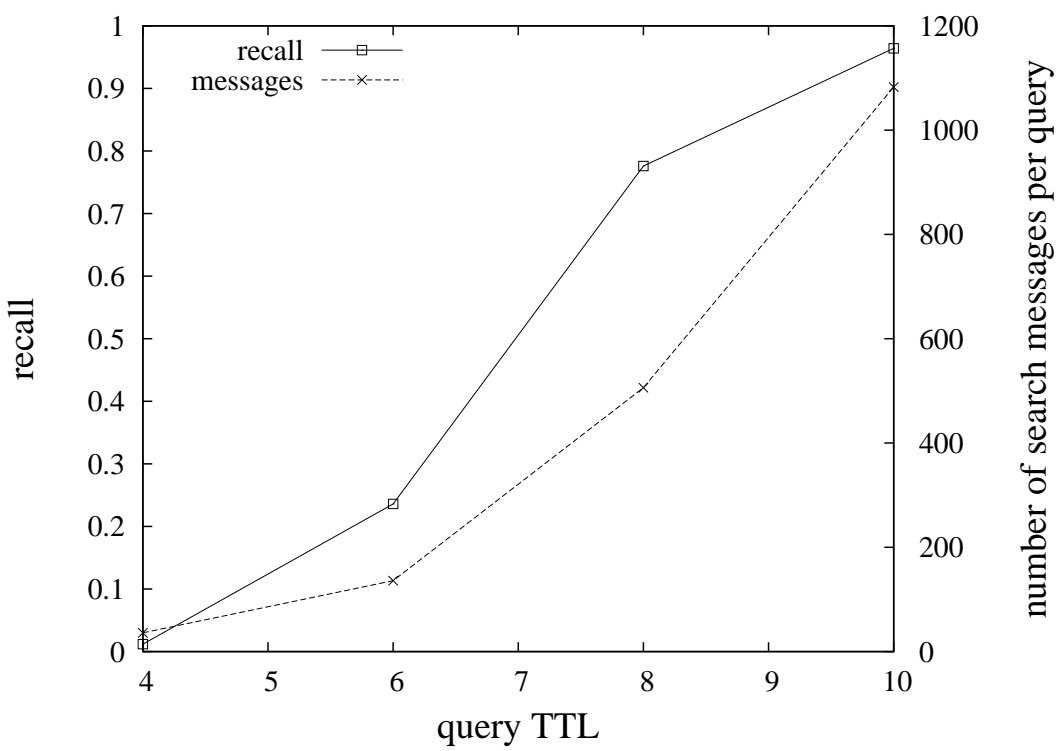

Fig. 4. Recall and query messages per query as a function of $t_{q}$.

incurred by both methods (i.e., almost 1,000 messages per query). When the network becomes organized, iCLUSTER (unlike [5]) achieves recall resembling that achieved by the flooding approach but for much less (up to 60\%) communication overhead. Finally, Fig. 6 indicates that, in terms of message traffic per query, flooding is the worst method.

In this set of experiments, iCLUSTER is compared with the peer clustering approach by Schmitz [5] and the standard exhaustive search approach by flooding, in terms of both communication load and retrieval accuracy. The experiments showed that iCLUSTER benefited the most from creating coherent clusters of peers with similar interests, as it resulted in high recall for much less network load than all its competitor methods. In particular, iCLUSTER can be almost as effective as flooding for much less message flow (i.e., the communication load reduced by approximately $70 \%$ ).

\section{Conclusion}

We present iCLUSTER, an approach for organizing peers into clusters and for supporting information retrieval functionality. iCLUSTER ensures clustering coherence while achieving high accuracy of retrievals by issuing a periodic rewiring procedure. The experimental results demonstrated that iCLUSTER outperforms other approaches for peer organization and retrieval, achieving higher clustering quality and higher recall for less communication overhead. Future work, includes experimentation with different query distributions, the study of the effect 


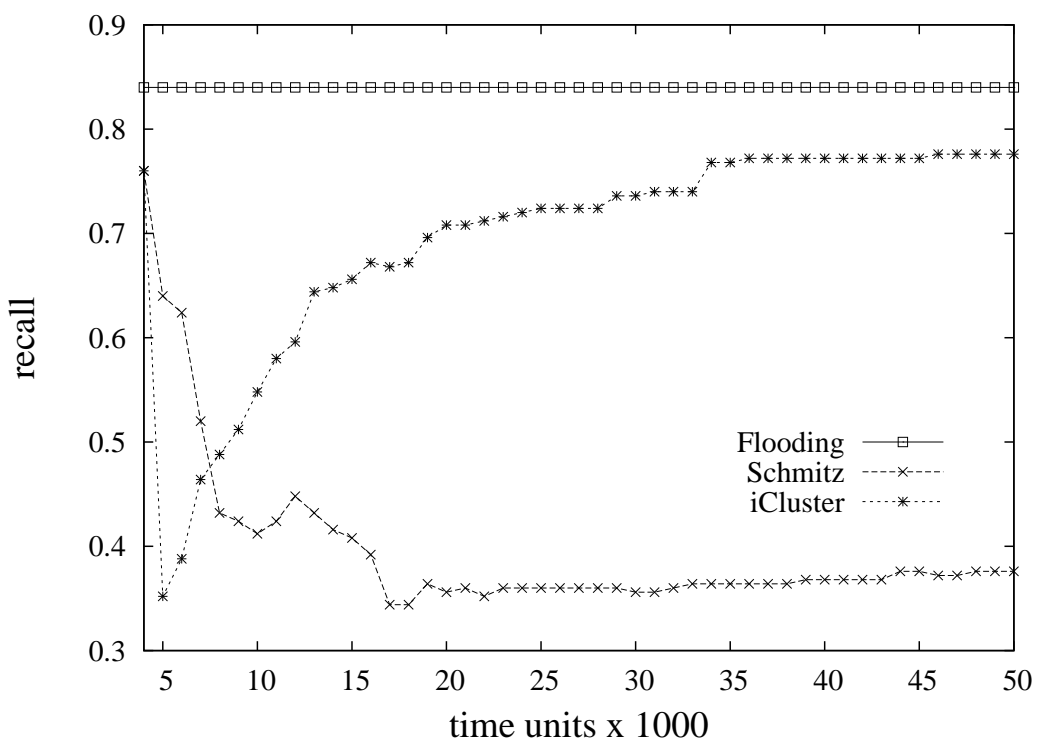

Fig. 5. Recall as a function of time for (a) iCluster (b) Flooding and (c) Schmitz [5].

of churn (dynamic peer insertions/deletions) to network organization and data retrieval, and extension of the proposed protocols to support both information retrieval and filtering functionality (publish/subscribe).

\section{Acknowledgements}

This work was funded by project "Herakleitos" of the Greek Secretariat for Research and Technology. We are grateful to Dr. Gerhard Weikum and the members of the Databases and Information Systems group of Max-Planck-Institute for their support.

\section{References}

1. Milojicic, D.S., Kalogeraki, V., Lukose, R., Nagaraja, K., Pruyne, J., Richard, B., Rollins, S., Xu, Z.: Peer-to-Peer Computing. Technical report, HP Labs (2002)

2. Stoica, I., Morris, R., Liben-Nowell, D., Karger, D.R., Kaashoek, M.F., Dabek, F., Balakrishnan, H.: Chord: A Scalable Peer-to-Peer Lookup Protocol for Internet Applications. IEEE/ACM Trans. on Networking 11(1) (2003)

3. Ratnasamy, S., Francis, P., Handley, M., Karp, R., Shenker, S.: A Scalable ContentAddressable Network. SIGCOMM '01

4. Crespo, A., Garcia-Molina, H.: Semantic Overlay Networks for P2P Systems. Technical report, Stanford Univ. (2003)

5. Schmitz, C.: Self-Organization of a Small World by Topic. P2PKM '04 


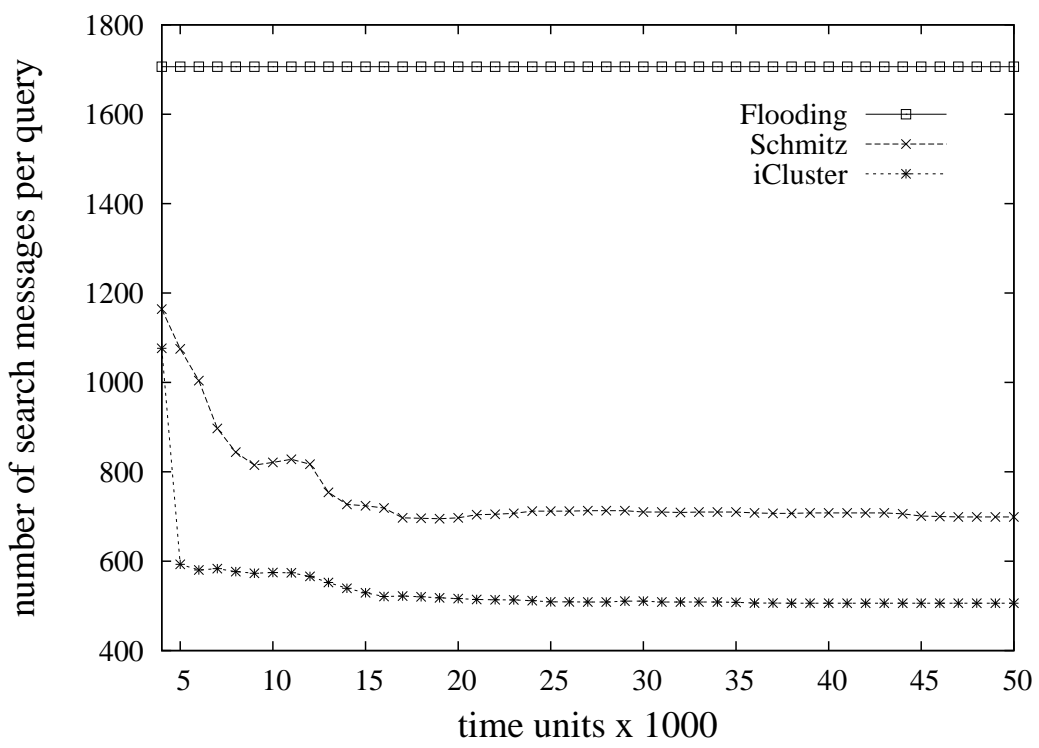

Fig. 6. Number of search messages as a function of time for (a) iCluster (b) Flooding and (c) Schmitz [5].

6. Loser, A., Tempich, C.: On Ranking Peers in Semantic Overlay Networks. WM '05

7. Spripanidkulchai, K., Maggs, B., Zhang, H.: Efficient Content Location using Interest-Based Locality in Peer-to-Peer Systems. INFOCOM '03

8. Nejdl, W., Wolf, B., Qu, C., Decker, S., Sintek, M., Naeve, A., Nilsson, M., Palmer, M., Risch, T.: EDUTELLA: a P2P Networking Infrastructure based on RDF. WWW'02

9. Decker, S., Schlosser, M., Sintek, M., Nejdl, W.: HyperCuP - Hypercubes, Ontologies and Efficient Search on P2P Networks. AP2PC '02

10. Lu, J., Callan, J.: Content-Based Retrieval in Hybrid P2P Networks. CIKM '03

11. Klampanos, I., Jose, J.: An Architecture for Information Retrieval over SemiCollaborating Peer-to-Peer Networks. ACM SAC '04

12. Parreira, J.X., Michel, S., Weikum, G.: p2pDating: Real Life Inspired Semantic Overlay Networks for Web Search. Inf. Proc. and Manag. 43(1) (2007)

13. Crespo, A., Garcia-Molina, H.: Routing Indices for P2P Systems. ICDCS '02

14. Li, M., Lee, W.C., Sivasubramaniam, A.: Semantic Small World: An Overlay Network for Peer-to-Peer Search. ICNP '04

15. Steinbach, M., Karypis, G., Kumar, V.: A Comparison of Document Clustering Techniques. TextDM '00

16. Hughes, D., Coulson, G., Walkerdine, J.: Free Riding on Gnutella Revisited: The Bell Tolls? IEEE DS Online 6(6) (2005)

17. Schmitz, C., Staab, S., Tempich, C.: Socialisation in Peer-to-Peer Knowledge Management. I-KNOW' 04

18. Hui, K., Lui, J., Yau, D.: Small-world Overlay P2P Networks: Construction, Mmanagement and Handling of Dynamic Flash Crowds. Computer Networks 50(15) (2006) 